\title{
Isolating the Modulatory Effect of Expectation on Pain Transmission: A Functional Magnetic Resonance Imaging Study
}

\author{
John R. Keltner, ${ }^{1}$ Ansgar Furst, ${ }^{2}$ Catherine Fan, ${ }^{2}$ Rick Redfern, ${ }^{2}$ Ben Inglis, ${ }^{2}$ and Howard L. Fields ${ }^{3}$ \\ ${ }^{1}$ Pain Management Center, Department of Anesthesia and Perioperative Care, University of California San Francisco, San Francisco, California 94143, \\ ${ }^{2}$ Henry H. Wheeler, Jr. Brain Imaging Center, Helen Wills Neuroscience Institute, University of California Berkeley, Berkeley, California 94608 , and \\ ${ }^{3}$ Wheeler Center for the Neurobiology of Addiction, Department of Neurology, University of California San Francisco, Emeryville, California 94608
}

\begin{abstract}
We use a novel balanced experimental design to specifically investigate brain mechanisms underlying the modulating effect of expected pain intensity on afferent nociceptive processing and pain perception. We used two visual cues, each conditioned to one of two noxious thermal stimuli $\left[\sim 48^{\circ} \mathrm{C}\right.$ (high) or $47^{\circ} \mathrm{C}$ (low) . The visual cues were presented just before and during application of the noxious thermal stimulus. Subjects reported significantly higher pain when the noxious stimulus was preceded by the high-intensity visual cue. To control for expectancy effects, for one-half of the runs, the noxious thermal stimuli were accompanied by the cue conditioned to the other stimulus. Comparing functional magnetic resonance imaging blood oxygenation level-dependent activations produced by the high and low thermal stimulus intensities presented with the high-intensity visual cue showed significant activations in nociceptive regions of the thalamus, second somatosensory cortex, and insular cortex. To isolate the effect of expectancy, we compared activations produced by the two visual cues presented with the high-intensity noxious thermal stimulus; this showed significant differences in the ipsilateral caudal anterior cingulate cortex, the head of the caudate, cerebellum, and the contralateral nucleus cuneiformis (nCF). We propose that pain intensity expectancy modulates activations produced by noxious stimuli through a distinct modulatory network that converges with afferent nociceptive input in the $\mathrm{nCF}$.
\end{abstract}

Key words: pain modulation; brainstem; cingulate; cuneiformis; insula; sensory cortex

\section{Introduction}

The intensity of perceived pain is a robust function of the intensity of the noxious stimulus that precedes it (Price, 1999). However, it is also strongly influenced by expectation (Hsieh et al., 1999; Ploghaus et al., 1999, 2003; Price et al., 1999; Petrovic et al., 2002; Porro et al., 2002; Jensen et al., 2003; Wager et al., 2004; Koyama et al., 2005), attention (Chua et al., 1999; Peyron et al., 1999; Longe et al., 2001; Bantick et al., 2002; Tracey et al., 2002; Villemure and Bushnell, 2002; Valet et al., 2004), and arousal (Chua et al., 1999; Peyron et al., 1999; Porro et al., 2003). Although expectation has been studied using functional imaging and psychophysical approaches (Price and Fields, 1997; Ploghaus et al., 2003), its contribution to the pain experience is incompletely understood.

The afferent nociceptive pathway projects via thalamic nuclei to somatosensory, insular, and anterior cingulate (ACC) cortices (Willis and Westlund, 1997; Casey and Bushnell, 2000; Craig,

\footnotetext{
Received 0ct. 19, 2005; revised March 16, 2006; accepted March 18, 2006.

This work was supported by United States Public Health Service Grant NS 21445, Pfizer, and the International Anesthesia Research Society.

Correspondence should be addressed to Dr. Howard L. Fields, Wheeler Center for the Neurobiology of Addiction, University of California San Francisco, 5858 Horton Street, Suite 200, Emeryville, CA 94608. E-mail: hlf@phy.ucsf.edu.

DOI:10.1523/JNEUROSCI.4463-05.2006

Copyright $\odot 2006$ Society for Neuroscience $\quad$ 0270-6474/06/264437-07\$15.00/0
}

2003). Functional imaging confirms that these sites are activated by noxious stimuli in humans (Casey, 1999; Peyron et al., 1999; Casey and Bushnell, 2000). However, imaging studies typically use repeated or prolonged noxious stimuli, which not only elicit pain but also produce changes in expectation, attention, and arousal. These changes introduce a potential confound because the brain activation patterns observed could reflect either the noxious stimulus intensity or the expectancy of pain (Petrovic et al., 2002; Tracey et al., 2002; Valet et al., 2004; Wager et al., 2004; Zambreanu et al., 2005). In fact, pain-predictive cues alone can activate the anterior cingulate and insular cortices, enhance the cortical activation produced by innocuous stimuli, and increase reported pain intensity (Sawamoto et al., 2000; Ploghaus et al., 2003; Koyama et al., 2005).

One possible mechanism for the modulatory effect of expectancy on pain is that it activates descending modulatory systems (Fields, 2004; Fields et al., 2006). Imaging studies are consistent with this hypothesis. During noxious stimulation, expectation for reduced pain (i.e., placebo) activates the rostral ACC (rACC), medial prefrontal cortex (mPFC), and periaqueductal gray (PAG) (Bantick et al., 2002; Petrovic et al., 2002; Tracey et al., 2002; Valet et al., 2004; Wager et al., 2004). Furthermore, activations in rACC correlate with those in bilateral amygdala and PAG under the condition of reduced pain expectancy (Bingel et al., 2006). Anticipation of pain also activates the rACC, mPFC, and 
PAG (Chua et al., 1999; Hsieh et al., 1999; Ploghaus et al., 2001, 2003). These studies suggest that there is bidirectional descending control of pain by a pathway with input from limbic forebrain and amygdala that converges on the midbrain PAG and, acting through pontomedullary nuclei, controls spinal pain transmission neurons (Fields, 2004; Fields et al., 2006).

The objective of this experiment was to address how expectation modulates pain perception and to what extent such modulation is accompanied by activation of brain areas implicated in descending modulatory processes. We used a balanced design to isolate the contributions of stimulus intensity and expectation for pain to functional magnetic resonance imaging (fMRI) blood oxygenation level-dependent (BOLD) activation patterns induced by repeated noxious stimulation.

\section{Materials and Methods}

Participants. Twenty-seven volunteers (15 males and 12 females, 26 right-handed and 1 left-handed) aged 19-27 years entered the study after written informed consent. All of the subjects were students at The University of California at Berkeley. The protocol was approved by the Committee on Human Research at the University of California at San Francisco as well as by the Committee for Protection of Human Subjects at the University of California at Berkeley.

Pain stimulus and subjective pain ratings. The noxious thermal stimuli were produced using a $3 \times 3 \mathrm{~cm}$ Peltier Thermode (Medoc, Ramat Yishay, Israel). The subject rested his/her left hand on the thermode in such a way that the subject had the freedom to remove his/her hand if the thermal stimulus was too uncomfortable. To limit the total amount of thermal energy delivered onto each subject's hand and to minimize sensitization effects, the heat stimulation was delivered onto six different $3 \times$ $3 \mathrm{~cm}$ regions of the palm on the left hand. Each $3 \times 3 \mathrm{~cm}$ region was subjected to a maximum of $5 \mathrm{~min}$ of thermal stimulation in the range of $47-48^{\circ} \mathrm{C}$ and $5 \mathrm{~min}$ of $33^{\circ} \mathrm{C}$ baseline stimulation. For each region, the thermal stimulations were divided into blocks lasting $30 \mathrm{~s}$. In between the $30 \mathrm{~s}$ blocks were $4 \mathrm{~s}$ intervals during which the temperature changed at a rate of change of $3.5-3.75^{\circ} \mathrm{C}$ per second.

Out of concern that repeated rating of the noxious thermal stimuli would itself alter the subject's experience or ratings of pain (Levine et al., 1982), we performed two separate experiments. The first, outside the magnet, involved 11 subjects ( 6 males and 5 females, all right-handed) performing the psychophysical protocol. One subject was excluded because that individual's verbal pain rating (VPR) of 9 was at $42^{\circ} \mathrm{C}$, which is significantly below the threshold for pain in the vast majority of subjects. The psychophysical protocol was the same as that used for the imaging study, except that the VPR was obtained for every thermal stimulus. Verbal pain reports were obtained only in the last $5 \mathrm{~s}$ of each thermal stimulus. In the second experiment, inside the magnet, 16 subjects ( 9 males and 7 females, 15 right-handed and 1 left-handed) underwent the pain-expectation protocol but were only asked to rate the noxious thermal stimuli between imaging acquisition runs and only for calibration purposes (described below). None of the 11 subjects in the psychophysical experiment participated in the imaging experiment. To reduce the potential stress of a novel and restricted environment, each subject underwent a brief training session in the magnet, without noxious stimulation, before the imaging procedure. Data sets from three additional subjects were excluded from the imaging analysis because of clear motion artifacts.

Pain expectation protocol. Our experiments were designed to address two goals: (1) to confirm expectancy effects on reports of subjective pain experience elicited by the noxious thermal stimuli that we used for the imaging study, and (2) to determine how pain predictive cues modulate BOLD responses to noxious thermal stimuli.

We explicitly and independently manipulated both the actual intensity of the noxious stimulus and the subject's expectation of intensity. Expectation was manipulated by training subjects with two distinct visual cues (red or blue with verbal instructions) explicitly paired with either a highor low-intensity noxious stimulus. The high pain intensity cue consisted of the message "High Temperature" printed in a white font in the center of the screen with a red background filling the rest of the visual field. The low pain intensity visual cue consisted of the message "Low Temperature" printed in a white font in the center of the screen with a blue background filling the rest of the visual field. We used both conditioning and verbal instruction because both have been demonstrated to have robust and independent effects on expectancy (Voudouris et al., 1990; Stewart-Williams and Podd, 2004). After training, each level of noxious stimulus was preceded by the high- or low-intensity cue.

The design of the current study differs from that used in previous studies of expectation in that it is balanced: we used visual cues conditioned to high and low noxious thermal stimuli to elicit expectations that were for either higher or lower (but still significant) levels of pain. Following conditioning, these two cues preceded noxious thermal stimuli of higher or lower intensity. This design brings the factor of expected pain intensity under experimenter control and provides an improved method for minimizing processes such as anxiety, fear, arousal and attention (Ploghaus et al., 2003).

Our protocol involved three separate steps: calibration, conditioning, and experiment.

Step 1: calibration. We established an individual verbal report metric (Coghill et al., 2003) for each subject for the noxious thermal temperature range used in our experiment $\left(44-48^{\circ} \mathrm{C}\right)$. During the calibration task, the presentation of the temperatures was either SERIES 1: 44, 47, 45, $46,48^{\circ} \mathrm{C}$; or SERIES 2: $X, X+0.5, X+1, X+0.5, X, X+1{ }^{\circ} \mathrm{C}$, where $X$ was the temperature from SERIES 1 that received a VPR score of 7-9 (other than $48^{\circ} \mathrm{C}$ ). The subject was instructed to rate each thermal stimulus based on a $1-10$ verbal pain rating scale (increments of 0.5 ), where 1 represented no pain, and 10 reflected the (theoretical) level of perceived pain intensity (not pain unpleasantness) that would induce the subject to remove his/her palm from the thermode. For each subject, the temperature corresponding to a verbal report of 9 from the verbal pain report metric was designated as the high-temperature stimulus, whereas a temperature $1^{\circ} \mathrm{C}$ lower was designated as the low-temperature stimulus. In the imaging experiment, the calibration was performed on the gurney but at the mouth of the magnet.

Step 2: conditioning. After the initial calibration, the subject was positioned inside the magnet, the Peltier thermode was applied to a second $3 \times 3 \mathrm{~cm}$ region of the palm, and each subject was conditioned using his/her individualized high- and low-temperature stimulations along with a high-pain visual cue and a low-pain visual cue. During the conditioning period, five pairs of high-intensity cue and high-intensity noxious thermal stimulation were interleaved with five pairs of low-intensity cue and lower level noxious thermal stimulation.

Step 3: psychophysical and imaging experiments. Following the conditioning protocol, we studied the effect of the cues on subsequent pain reports and neural processing of a standard painful stimulus. We predicted that the visual cue associated with lower pain intensities during conditioning would induce reports of relatively lower pain intensity, whereas cues predictive of higher thermal intensities would be associated with greater pain intensity. This design allows us to observe the subject's brain activity when the thermal stimulus intensity is constant, but the expectancy level is varied. Because of the balanced crossover design, we were also able to observe the subject's pattern of brain activation when the thermal stimulus changes but the predictive cue for intensity is unchanged.

Forty stimulations (10 high-temperature stimuli paired with hightemperature cues, 10 high-temperature stimuli paired with low-temperature cues, 10 low-temperature stimuli paired with high-temperature cues, and 10 low-temperature stimuli paired with low-temperature cues) were presented in a random order. Each of the 10 stimulations was applied to one of the four remaining $3 \times 3 \mathrm{~cm}$ region of the left palm. During the forty stimulations 340 echoplanar acquisitions were obtained.

Each cue and thermal stimulation pair consisted of $30 \mathrm{~s}$ of baseline temperature stimulation $\left(33^{\circ} \mathrm{C}\right) ; 4 \mathrm{~s}$ for the Peltier thermode to ramp from the baseline to the low or high noxious thermal stimulation temperature; $30 \mathrm{~s}$ of the low or high stimulation temperature; $4 \mathrm{~s}$ for the Peltier thermode to ramp down from the painful stimulation temperature to the baseline. Note that the cue presentation remained on through- 
out the last $6 \mathrm{~s}$ of baseline temperature stimulation, the two ramp periods, and during the $30 \mathrm{~s}$ noxious thermal stimulation period. The duration of a single cycle of baseline to painful temperature and return to baseline was $68 \mathrm{~s}$. Both psychophysical and imaging studies were of $\sim 40$ min in duration, and four stimulus intensity recalibrations were performed during each individual psychophysical or imaging study.

When thermal stimuli of constant intensity are repeatedly applied to the skin, they can produce highly variable subjective pain reports. This variability can be attributable to either psychological factors (attention, expectation, anxiety) or to sensitization or desensitization of peripheral or central afferent nociceptive pathways (Price et al., 1977). To minimize the potential effect of sensitization, desensitization and summation, the high-level thermal stimulus was adjusted in all subjects at $10 \mathrm{~min}$ intervals with the intent to produce a stable pain rating of 9 . The perceived high-intensity noxious thermal stimulus from the previous $10 \mathrm{~min}$ run was rated using the verbal pain rating. If the rating was 9 , the temperature of the thermal stimulus was not changed. If the rating was 10 , the temperature of the thermal stimulus was decreased $0.1^{\circ} \mathrm{C}$. If the rating was 8 , the temperature was increased 0.1 up to $48.0^{\circ} \mathrm{C}$. If the rating was $<8$, then the temperature was increased 0.2 up to $48.0^{\circ} \mathrm{C}$. In general, under the conditions of this experiment, the perceived pain intensity to the highintensity stimulus declined, requiring slight increases in thermode peak temperature and rate of rise. Over the $40 \mathrm{~min}$ course for each of the 16 imaging subjects, the high-intensity stimulus temperature was, on average, increased $<0.025^{\circ} \mathrm{C}$ for each adjustment. Four of the 16 subjects required no adjustments for any of the high-intensity stimulus temperatures. Despite adjusting the temperature of the high-pain thermal stimulus, the maximum temperature used in each of the 16 experiments was never adjusted to $>48^{\circ} \mathrm{C}$. Consequently, the average rating for the 32 $48^{\circ} \mathrm{C}$ stimulations used in the 16 imaging experiments was 8.1 , which was less than our goal of 9 .

The high and low noxious stimulation temperatures were separated by $1^{\circ} \mathrm{C}$ to increase the influence of the expectancy cues used in this experiment. Data from the calibration process indicated that accurately identifying the higher of two temperatures separated by $1^{\circ} \mathrm{C}$ can be difficult. When two different temperatures in the calibration process were separated by $1^{\circ} \mathrm{C}, 25 \%$ of the time the VPR score picked by the subject gave the two temperatures the same rating or rated the lower intensity stimulus higher on the VPR. The subjects' frequent failure to accurately discriminate noxious thermal stimuli separated by $1^{\circ} \mathrm{C}$ is consistent with pilot studies of the subjective experience of the authors, which indicated that temperatures separated by $1^{\circ} \mathrm{C}$ can be difficult to differentiate during the course of the $40 \mathrm{~min}$ experiment and suggests that our goal of minimizing factors such as attention, arousal, and anxiety was at least partially achieved.

Data acquisition. A 4 tesla whole-body Varian (Palo Alto, CA) Inova MR scanner at the Wheeler Brain Imaging Center at the University of California Berkeley, Department of Psychology was used for these experiments. A whole-head quadrature transverse electromagnetic radiofrequency coil (MR Instruments, Minneapolis, MN) was used for image acquisition. A locally constructed bite bar to limit head movement was attached to this coil. The bite bar had a quick-release mechanism so that the subject could exit the magnet at his/her desire.

Oblique angle $\left(\sim 30^{\circ}\right.$ relative to the $z$-axis) two-shot echoplanar images (EPIs) were acquired with an effective repetition time (TR) of 2000 $\mathrm{ms}$, echo time of $22 \mathrm{~ms}$ for BOLD contrast, and a flip angle of $20^{\circ}$ to minimize inflow effects. The in-plane matrix was $64 \times 64$ voxels over a field of view of $22 \times 22 \mathrm{~cm}$. Twenty-two slices with a $4.5 \mathrm{~mm}$ thickness and slice gap of $0.5 \mathrm{~mm}$ were acquired from below the pons out to the olfactory cortex. The brainstem and prefrontal cortex were prioritized in our slice selection over somatosensory cortex. Three hundred forty (the first four scans were discarded to account for magnetic saturation) multislice volumes were acquired for each functional time series. Echoplanar functional images were coregistered to a high-resolution $(256 \times 256$ matrix) T1-weighted gradient echo multislice (GEMS) image volume covering the same slice positions and field-of-view. Last, T1-weighted three-dimensional $256 \times 256 \times 128$ vol magnetization-prepared fast low angle shot three-dimensional (MPFLASH 3D) were acquired to enable post hoc normalization of the data.
Table 1. Average verbal pain ratings in the psychophysics experiment outside the scanner

\begin{tabular}{lll}
\hline & High temperature & Low temperature \\
\hline High expectation & $8.20(0.84)$ & $6.42(1.32)$ \\
Low expectation & $7.37(1.20)$ & $5.71(1.35)$ \\
\hline
\end{tabular}

SDs are shown in parentheses.

Preprocessing and statistical modeling. fMRI data were reconstructed using in-house software that applied a linear-time interpolation algorithm to adjacent half $k$-space shots (resulting in an effective TR of $1 \mathrm{~s}$ ) and a temporal sync interpolation to correct for between-slice timing differences in image acquisition. All subsequent preprocessing was conducted in SPM2 (http://www.fil.ion.ucl.ac.uk/spm/software/spm2/). EPI scans were realigned to the first volume, motion-corrected using a sixparameter rigid-body transformation, and spatially smoothed applying an isotropic $8 \mathrm{~mm}$ full-width at half-maximum Gaussian kernel (firstlevel analyses only). Inspection of the movement parameters indicated no movement larger than $3 \mathrm{~mm}$ in any direction. MPFLASHs were coregistered to GEMS and normalized to the T1 Montreal Neurological Institute template $(2 \times 2 \times 2)$ using an affine 12 parameter transformation with nonlinear deformations. The resulting normalization parameters were then applied to the EPI data. Talairach coordinate approximations (Talairach and Tournoux, 1988) were determined with Matthew Brett's mni2tal routine (http://www.mrc-cbu.cam.ac.uk/Imaging/Common/ mnispace.shtml). Anatomical regions were identified with the Tailarach Daemon (http://ric.uthscsa.edu/projects/talairachdaemon.html) using a cube search range of $3 \mathrm{~mm}$.

fMRI data were modeled within an event-related block design using the Generalized Linear Model (GLM) within SPM2. The onsets for the four experimental conditions (High Expectation and High Temperature, High Expectation and Low Temperature, Low Expectation and High Temperature, Low Expectation and Low Temperature) were modeled as 30 s epochs of sustained neural activity starting at the onset of the noxious stimulus (see Fig. 1, diagram). Baseline activity was explicitly modeled averaging the $24 \mathrm{~s}$ periods between trials in which participants rested (this excluded thermode ramp on and off phases). The resulting boxcar functions were convolved with a canonical hemodynamic response function. To account for the serial autocorrelation inherent to fMRI series the $\mathrm{AR}(1)$ plus whitening algorithm was applied. Low-frequency drifts were removed by applying a temporal high-pass filter (cutoff, 1/100 Hz). Assuming intersubject variance homogeneity, the results of the first-level analyses (GLMs) were subsequently combined in a full-brain, voxelwise second-level random-effects analysis. We compared the BOLD activation in each experimental condition to baseline (see Table 2, Fig. 1). Furthermore, we performed two more specific comparisons contrasting high versus low expectation when the temperature was high and high versus low temperature when the expectation was high (see Table 2, Figs. 2,3 ). Group level $t$ statistics ( $\mathrm{df}=15$; uncorrected $p<0.001$ ) were computed for each contrast and thresholded at a minimum cluster size of 10 voxels. Local maxima and anatomical locations for relevant contrasts are reported in Table 2 .

\section{Results}

\section{Psychophysics}

A two-way repeated measures ANOVA of the VPR scores with the factors expectation (high, low) and temperature (high, low) was conducted. This revealed a main effect for expectation $\left(F_{(1,10)}=\right.$ $13.8 ; p<0.005)$ confirming that participants rated a given temperature consistently as more painful when they were expecting a high-intensity noxious stimulus (Table 1 ). We also observed a main effect for temperature $\left(F_{(1,10)}=63.8, p<0.0001\right)$ indicating that high-intensity noxious stimuli were rated as significantly more painful compared with low-intensity stimuli (Table 1). However, there were no interactions. 
Activation in afferent pain circuits

The afferent pain circuit consists of primary afferent nociceptors, dorsal horn neurons, including ascending projections to brainstem and thalamic nuclei that project to insular, somatosensory, and anterior cingulate cortex (Bushnell and Apkarian, 2005). Consistent with previous reports, we observed significant activations compared with baseline in nociceptive regions of the thalamus, insular cortex, somatosensory cortex, and anterior cingulate cortex. This pattern was observed when the high-intensity noxious stimulus was preceded and accompanied by the high-intensity cue (Fig. 1, Table 2). Under this condition, significant activations in the orbital frontal cortex, amygdala, ventral striatum, and the brainstem nucleus cuneiformis (nCF) were also observed. Similar to the VPR, fMRI BOLD activations were significantly less when the high-intensity noxious stimulus was preceded and accompanied by the lowintensity visual cue (Fig. 1). As expected, when preceded by the high-intensity visual cue, the lower intensity noxious stimulus elicited markedly reduced activation (compared with baseline) compared with the high-intensity stimulus Figure 1.

\section{Isolating the effect of pain}

predictive cues

These observations clearly demonstrate the very significant contribution of expectancy to the magnitude of AMRI BOLD signals produced by cutaneous noxious thermal stimuli. To further analyze the separate contributions of expectancy and stimulus intensity, we made two different fMRI BOLD activation comparisons; first for the two different expectancy levels (intensity cues) when the noxious stimulus intensity was the same and, second, for the two noxious stimulus intensities when the expectation level (intensity cue) was the same.

To isolate the expectancy effect, we studied the high noxious stimulus intensity condition and compared high-intensity expectancy (red cue) and low-intensity expectancy (blue cue). This analysis revealed that the $\mathrm{ACC}$, cerebellum, and the dorsolateral pontomesencephalic region (nCF) (Zambreanu et al., 2005) have increased BOLD activation when expectancies are for higher pain intensity (Fig. 2). Apparently, the expectancy effect requires a higher noxious thermal stimulus, as evidenced by the fact that when activations produced by the two expectation levels were contrasted under the low noxious stimulus condition, no significant differences in BOLD activations were found.

We also examined the pattern of activation produced by a relatively small increase in noxious thermal stimulus intensity $\left(1^{\circ} \mathrm{C}\right)$ when expectancy was controlled. When the visual cue predicted the high-intensity stimulus, the contrast between high and low thermal stimulus intensity (Fig. 3 ) revealed activations in the nociceptive regions of the thalamus, second somatosensory cortex, and the contralateral insula. It is important to emphasize that this is a temperature difference of $1^{\circ} \mathrm{C}$ at most, a difference that many of our subjects could not consistently distinguish. Further- more, when this same noxious stimulus intensity contrast was computed for the low-intensity visual cue, no significant fMRI BOLD activation was found.

\section{Discussion}

In the current study, we used a balanced experimental design with two levels of noxious thermal stimulation and two corresponding levels of expectancy produced by visual cues briefly conditioned with each of the two noxious stimuli. By explicitly manipulating expectancy while minimizing factors such as attention, arousal, and anxiety, we were able to extend current understanding of its robust contribution to perceived pain intensity. It is likely that most fMRI studies and particularly those that employ repeated noxious stimulation have an implicit expectancy component. Previous work has shown that expectancy for pain (versus no pain) enhances the perceived intensity of a somatosensory stimulus (Benedetti et al., 1997; Price, 1999; Sawamoto et al., 2000) and that expectancy for reduced pain (e.g., placebo) can reduce the pain reported in response to a noxious stimulus (Voudouris et al., 1990; Price et al., 1999; Colloca and Benedetti, 2005; Hoffman et al., 2005; Koyama et al., 2005). Here, we confirm and extend those studies by demonstrating that the level of expected pain intensity significantly alters perceived pain intensity when the comparison is between two noxious thermal stimuli of slightly different intensity. Significantly, consistent with the psychophysical data, our imaging results indicate that expectation and noxious stimulus intensity act in an additive manner on afferent pathways activated by cutaneous noxious thermal stim- 
Table 2. Peak BOLD activations for key comparisons

\begin{tabular}{|c|c|c|c|c|c|c|c|c|c|}
\hline \multirow[b]{2}{*}{ Contrast } & \multirow[b]{2}{*}{ Anatomical region } & \multirow[b]{2}{*}{ Brodmann's area } & \multicolumn{3}{|c|}{ MNI coordinates } & \multicolumn{3}{|c|}{ Talairach coordinates } & \multirow[b]{2}{*}{$t$ value } \\
\hline & & & $x$ & $y$ & $z$ & $x$ & $y$ & $Z$ & \\
\hline \multirow[t]{12}{*}{ HiE_HiT versus baseline (Fig. 1) } & Left transverse temporal gyrus/insula & BA 41/BA 13 & -40 & -26 & 14 & -40 & -25 & 14 & 4.69 \\
\hline & Right superior temporal gyrus & BA 38 & 44 & 14 & -14 & 44 & 13 & -12 & 6.52 \\
\hline & Left medial frontal gyrus & BA 8 & -2 & 36 & 44 & -2 & 37 & 39 & 5.74 \\
\hline & Left superior frontal gyrus & BA 10 & -22 & 62 & 16 & -22 & 61 & 12 & 5.11 \\
\hline & Right insula & BA 13 & 40 & -14 & 18 & 40 & -13 & 17 & 5.48 \\
\hline & Left inferior frontal gyrus & BA 9 & -44 & 8 & 32 & -44 & 9 & 29 & 5.48 \\
\hline & Right nucleus cuneiformis & $\mathrm{N} / \mathrm{A}$ & 8 & -28 & -22 & 8 & -28 & -17 & 4.05 \\
\hline & Left middle frontal gyrus & BA 6 & -30 & -10 & 50 & -30 & -7 & 46 & 4.01 \\
\hline & Left cerebellum & $\mathrm{N} / \mathrm{A}$ & -32 & -60 & -38 & -32 & -60 & -29 & 5.31 \\
\hline & Left cingulate gyrus & BA 24 & -2 & 12 & 36 & -2 & 13 & 32 & 4.82 \\
\hline & Left caudate & N/A & -8 & 4 & 4 & -8 & 4 & 3 & 4.74 \\
\hline & Left thalamus & $\mathrm{N} / \mathrm{A}$ & -6 & -22 & 16 & -6 & -21 & 16 & 5.07 \\
\hline \multirow[t]{4}{*}{ HiE_HiT versus LoE_HiT (Fig. 2) } & Left cingulate gyrus & BA 24 & -4 & -6 & 42 & -4 & -4 & 39 & 5.49 \\
\hline & Left medial frontal gyrus & BA 8 & -2 & 32 & 46 & -2 & 33 & 41 & 5.24 \\
\hline & Right cerebellum & $\mathrm{N} / \mathrm{A}$ & 12 & -62 & -18 & 12 & -61 & -12 & 5.91 \\
\hline & Right nucleus cuneiformis & $\mathrm{N} / \mathrm{A}$ & 10 & -28 & -18 & 10 & -28 & -14 & 4.45 \\
\hline \multirow[t]{3}{*}{ HiE_HiT versus HiE_LoT (Fig. 3) } & Insula/transverse temporal gyrus & BA $13 / 41$ & -38 & -28 & 14 & -38 & -26 & 14 & 3.94 \\
\hline & Right thalamus & $\mathrm{N} / \mathrm{A}$ & 10 & -18 & 12 & 10 & -17 & 12 & 4.33 \\
\hline & Superior temporal gyrus & BA 22 & 54 & 4 & 2 & 53 & 4 & 2 & 3.91 \\
\hline
\end{tabular}

HiE_HiT, High expectation and high temperature; HiE_LoT, high expectation and low temperature; LoE_HiT, low expectation and high temperature; N/A, not applicable; BA, Brodmann's area.

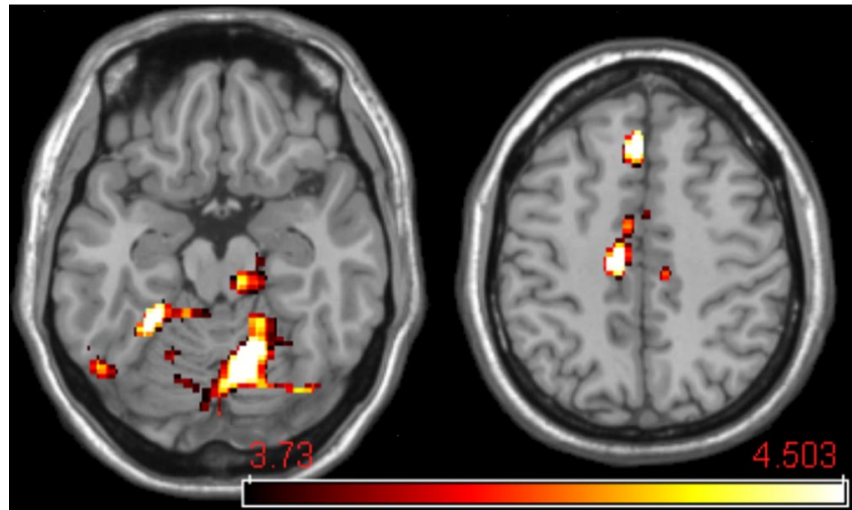

Figure 2. Expectation-associated BOLD activation. To identify brain regions participating in expectation, the expectancy effect was isolated. Using the high-temperature stimulus $\left(\sim 48^{\circ} \mathrm{C}\right.$ ) condition, we compared high-intensity expectancy (red cue) and low-intensity expectancy (blue cue). The $\mathrm{CACC}$, cerebellum, and the dorsolateral pontomesencephalic region including the $\mathrm{nCF}$ have increased BOLD activation when expectancies are for higher pain intensity.

uli. The current study provides explicit support for the hypothesis that expectancy for high pain intensity is necessary for maximal activation of afferent pain circuitry and maximal perceived pain intensity. A corollary is that neither high stimulus intensity nor high levels of expectation alone are typically sufficient to produce maximal activation of afferent pain circuitry and maximal perceived pain intensity.

Previous work on distraction and expectation has shown that activation in the afferent nociceptive circuit tracks reported pain intensity. For example, expectation for pain (versus no pain) correlates with increased activation in the cACC (Sawamoto et al., 2000) and S1 (Porro et al., 2002). Conversely, activation of cACC, insula, S1/S2, and thalamus by noxious stimulation is inhibited by distraction (Longe et al., 2001; Bantick et al., 2002; Valet et al., 2004) and by reduced pain expectation (Wager et al., 2004; Koyama et al., 2005).

What is the neural mechanism by which expectancy produces these robust effects on afferent pain circuitry and reported pain

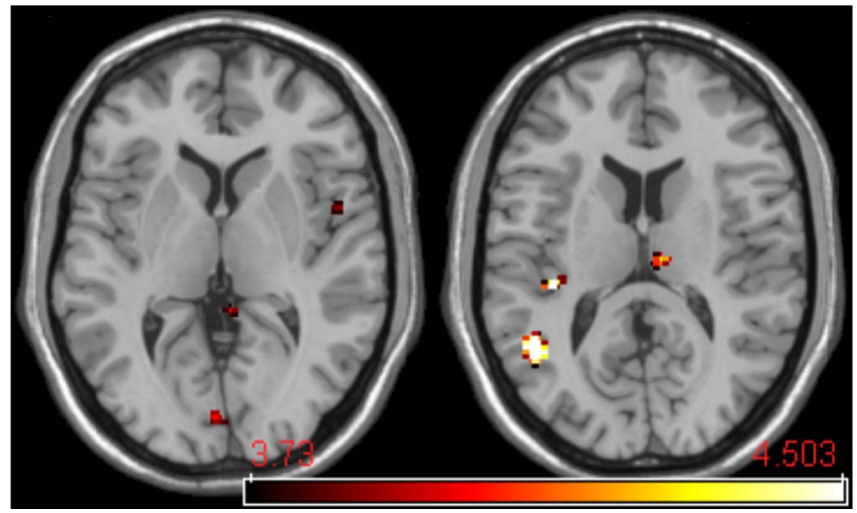

Figure 3. Thermal stimulus intensity-associated BOLD activation. To identify brain regions participating in the discrimination of noxious thermal stimuli, a small increase in noxious thermal stimulus intensity $\left(1^{\circ} \mathrm{C}\right)$ was applied while the level of expectancy (predictive cue) was maintained. When the visual cue predicted the high-intensity stimulus (red cue), the BOLD contrast between high and low thermal stimulus intensity revealed activations in the nociceptive regions of the thalamus, second somatosensory cortex, and the contralateral insula.

intensity? Functional imaging studies have begun to address this issue, and there is emerging, although still incomplete evidence that at least part of the expectancy effect is mediated by descending pain modulatory circuits. For example, distraction or expectancy for either increased or decreased pain perception is associated with activation of brain regions proposed to contribute to descending pain modulatory circuits. These regions include the medial prefrontal cortex [encompassing the rACC and ventromedial prefrontal cortex (vmPFC)], the orbital and insular cortices, the amygdala, and the PAG (Bandler and Shipley, 1994). During noxious stimulation, descending pain modulatory circuit elements including rACC, mPFC, and PAG are activated by distraction (Bantick et al., 2002; Tracey et al., 2002; Valet et al., 2004) and by expectation for reduced pain (i.e., placebo analgesia) (Petrovic et al., 2002; Wager et al., 2004; Koyama et al., 2005). Importantly, anticipation of pain (versus no pain) activates the 
insula, rACC, vmPFC, cerebellum, and PAG (Chua et al., 1999; Hsieh et al., 1999; Ploghaus et al., 2001, 2003).

Our data indicate that neural activity elicited by expectancy and noxious stimulus sum to increase reported pain intensity and to enhance activation of afferent pain circuitry at the thalamic and cortical level. Where does this summation occur? One possibility is the dorsal horn. The well described pain modulatory system that descends from limbic neocortex, amygdala, and hypothalamus via the midbrain PAG and nucleus cuneiformis to the rostral ventral medial medulla exerts direct control over dorsal horn pain transmission neurons (Fields et al., 2006). In fact, a recent fMRI connectivity analysis demonstrated that placebo analgesia-dependent activations of rACC are correlated with activity in the amygdala and the PAG (Bingel et al., 2006). Although most studies have emphasized descending inhibition, it is important that, in the current context, this same descending pathway can exert either inhibitory or facilitatory influences on ascending pain pathways (Fields, 2004; Fields et al., 2006).

Although we did not observe activations in PAG or medulla, we did find that, for a high-level noxious stimulus, high pain expectancy increased activation in the region of the cACC, the cerebellum, and the nCF (Fig. 2). The cACC projects directly to the PAG (Hardy and Leichnetz, 1981) and, in primates, neurons in this area respond to both noxious stimuli and to cues predictive of noxious stimuli (Koyama et al., 1998). Pain expectancy has also been reported to produce enhancement of cACC activity and reports of pain in the presence of an innocuous warm stimulus (Sawamoto et al., 2000). Ploghaus et al. (1999) compared the effect of visual predictive cues on responses to noxious heat and innocuous warm stimuli. During the noxious heat preceded by a cue for noxious heat, they observed significant $\mathrm{AMRI} B O L D$ activations in insular cortex as well as in the mid ACC and cerebellum that overlapped with the activations we observed under these conditions. Because they did not observe activations in these areas to the visual cues alone, they attributed the ACC and cerebellar activations to the noxious stimulus alone. Our data indicate that these midcingulate and cerebellar activations require both predictive information (expectancy) as well as the noxious stimulus itself.

The nCF is also of potential importance for pain modulation. Zambreanu et al. (2005) showed that nCF is activated by repeated noxious stimuli in humans under conditions of sensitization. Our data suggest that the activation they observed in nCF is attributable in part to an expectancy state that developed because of repeated noxious stimulation. The region of the $\mathrm{nCF}$ is a possible site of convergence of descending and ascending influences on pain, because, like the dorsal horn, it participates in both ascending transmission and in modulatory control of nociceptive input. $\mathrm{nCF}$ receives a massive afferent input from presumed nociceptive lamina I dorsal horn neurons (McMahon and Wall, 1985; Wiberg et al., 1987; Yezierski, 1988). In addition, nCF has cytoarchitecture similar to the PAG and projects directly to the rostral ventral medulla (RVM), which exerts direct bidirectional control of dorsal horn nociceptive neurons (Zambreanu et al., 2005; Fields et al., 2006). Furthermore, in the primate, the nCF receives massive input from the PAG, which in turn has inputs from Brodmann's area 24 (rACC), the hypothalamus, and the amygdala (Mantyh, 1982, 1983). Finally, Dunckley et al. (2005) have shown that, with repeated noxious stimuli, the PAG, RVM, and nCF are activated and that the $\mathrm{nCF}$ and RVM activations are correlated. Although those authors emphasized the ascending nociceptive input to $\mathrm{nCF}$, our data highlight the importance of (expectancy-based) descending facilitatory inputs from the limbic forebrain. To- gether, these findings suggest that the $\mathrm{nCF}$ is a site at which descending inputs from cortical regions activated by expectancy converge with and control ascending nociceptive projections from spinal cord to thalamus and hence to cortical receiving areas. In this regard, it is important to point out that, in rodents, both the nCF and the RVM have distinct but anatomically overlapping populations of modulatory neurons: off cells that inhibit and on cells that facilitate nociceptive transmission (Haws et al., 1989; Fields et al., 2006). This is consistent with the idea that descending modulatory circuits can either enhance or inhibit pain transmission.

In summary, comparing the effect of predictive cues specific to two different levels of noxious stimulus intensity revealed that expectation and noxious peripheral stimuli exert additive effects on afferent pathways underlying pain perception. This paradigm also revealed increased activation of the dorsolateral pontomesencephalic region, including the nucleus cuneiformis, contralateral to the noxious stimulus. These activations were maximal when the noxious thermal stimulus was preceded by a cue indicating a more intense noxious stimulus. This result is consistent with the hypothesis that expectation for a higher intensity noxious stimulus increases subjectively experienced pain intensity in part through the action of a descending pathway that facilitates nociceptive transmission at and/or caudal to the region of the nCF.

\section{References}

Bandler R, Shipley MT (1994) Columnar organization in the midbrain periaqueductal gray: modules for emotional expression? Trends Neurosci 17:379-389.

Bantick SJ, Wise RG, Ploghaus A, Clare S, Smith SM, Tracey I (2002) Imaging how attention modulates pain in humans using functional MRI. Brain 125:310-319.

Benedetti F, Amanzio M, Casadio C, Oliaro A, Maggi G (1997) Blockade of nocebo hyperalgesia by the cholecystokinin antagonist proglumide. Pain 71:135-140.

Bingel U, Lorenz J, Schoell E, Weiller C, Buchel C (2006) Mechanisms of placebo analgesia: rACC recruitment of a subcortical antinociceptive network. Pain 120:8-15.

Bushnell MC, Apkarian AV (2005) Representation of pain in the brain. In: Wall and Melzack's textbook of pain, Chap 6 (McMahon SB, Koltzenburg M, eds), pp 107-124. New York: Elsevier, Churchill Livingstone

Casey KL (1999) Forebrain mechanisms of nociception and pain: analysis through imaging. Proc Natl Acad Sci USA 96:7668-7674.

Casey KL, Bushnell MC (2000) Pain imaging. Seattle: IASP.

Chua P, Krams M, Toni I, Passingham R, Dolan R (1999) A functional anatomy of anticipatory anxiety. NeuroImage 9:563-571.

Craig AD (2003) A new view of pain as a homeostatic emotion. Trends Neurosci 26:303-307.

Coghill RC, McHaffie JG, Yen YF (2003) Neural correlates of interindividual differences in the subjective experience of pain. Proc Natl Acad Sci USA 100:8538-8542.

Colloca L, Benedetti F (2005) Placebos and painkillers: is mind as real as matter? Nat Rev Neurosci 6:545-552.

Dunckley P, Wise RG, Fairhurst M, Hobden P, Aziz Q, Chang L, Tracey I (2005) A comparison of visceral and somatic pain processing in the human brainstem using functional magnetic resonance imaging. J Neurosci 25:7333-7341.

Fields H (2004) State-dependent opioid control of pain. Nat Rev Neurosci 5:565-575.

Fields HL, Basbaum AI, Heinricher MM (2006) Central nervous system mechanisms of pain modulation. In: Wall and Melzack's textbook of pain, Chap 7 (McMahon SB, Koltzenburg M, eds). New York: Elsevier, Churchill Livingstone.

Hardy SGP, Leichnetz GR (1981) Cortical projections to the Periaqueductal gray in the monkey: a retrograde and orthograde horseradish peroxidase study. Neurosci Lett 22:97-101.

Haws CM, Williamson AM, Fields HL (1989) Putative nociceptive modula- 
tory neurons in the dorsolateral pontomesencephalic reticular formation. Brain Res 483:272-282.

Hoffman GA, Harrington A, Fields HL (2005) Pain and the placebo: what we have learned. Perspect Biol Med 48:248-265.

Hsieh JC, Stone-Elander S, Ingvar M (1999) Anticipatory coping of pain expressed in the human anterior cingulate cortex: a positron emission tomography study. Neurosci Lett 262:61-64.

Jensen J, McIntosh AR, Crawley AP, Mikulis DJ, Remington G, Kapur S (2003) Direct activation of the ventral striatum in anticipation of aversive stimuli. Neuron 40:1251-1257.

Koyama T, Tanaka YZ, Mikami A (1998) Nociceptive neurons in the macaque anterior cingulate activate during anticipation of pain. NeuroReport 9:2663-2667.

Koyama T, McHaffie JG, Laurient PL, Coghill RC (2005) The subjective experience of pain: Where expectations become reality. Proc Natl Acad Sci USA 102:12950-12955.

Levine JD, Gordon NC, Smith R, Fields HL (1982) Post-operative pain: effect of extent of injury and attention. Brain Res 234:500-504.

Longe SE, Wise R, Bantick S, Lloyd D, Johansen-Berg H, McGlone F, Tracey I (2001) Counter-stimulatory effects on pain perception and processing are significantly altered by attention: an fMRI study. NeuroReport 12:2021-2025.

Mantyh PW (1982) Forebrain projections to the periaqueductal gray in the monkey, with observations in the cat and rat. J Comp Neurol 206:146-158.

Mantyh PW (1983) Connections of midbrain periaqueductal gray in the monkey. II. Descending efferent projections. J Neurophysiol 49:582-594.

McMahon SB, Wall PD (1985) Electrophysiological mapping of brainstem projections of spinal cord lamina I cells in the rat. Brain Res 333:19-26.

Petrovic P, Kalso E, Petersson KH, Ingvar M (2002) Placebo and opioid analgesia: imaging a shared neuronal network. Science 295:1737-1740.

Peyron R, Garcia-Larrea L, Gregoire MC, Costes N, Convers P, Lavenne F, Mauguiere F, Michel D, Laurent B (1999) Haemodynamic brain responses to acute pain in humans: sensory and attentional networks. Brain 122:1765-1780.

Ploghaus A, Tracey I, Gati JS, Clare S, Menon RS, Matthews PM, Rawlins JN (1999) Dissociating pain from its anticipation in the human brain. Science 284:1979-1981.

Ploghaus A, Narain C, Beckmann CF, Clare S, Bantick S, Wise R, Matthews PM, Rawlins JN, Tracey I (2001) Exacerbation of pain by anxiety is associated with activity in a hippocampal network. J Neurosci 21:9896-9903.

Ploghaus A, Becerra L, Borras C, Borsook D (2003) Neural circuitry underlying pain modulation: expectation, hypnosis, placebo. Trends Cogn Sci 7:197-200.

Porro CA, Baraldi P, Pagnoni G, Serafini M, Facchin P, Maieron M, Nichelli P (2002) Does anticipation of pain affect cortical nociceptive systems? J Neurosci 22:3206-3214.
Porro CA, Cettolo V, Francescato MP, Baraldi P (2003) Functional activity mapping of the mesial hemispheric wall during anticipation of pain. NeuroImage 19:1738-1747.

Price DD (1999) Psychological mechanisms of pain and analgesia. Seattle: IASP.

Price DD, Fields HL (1997) Toward a neurobiology of placebo analgesia. In: The placebo effect: an interdisciplinary exploration (Harrington A, ed), pp 93-117. Cambridge, MA: Harvard UP.

Price DD, Hu JW, Dubner R, Gracely RH (1977) Peripheral suppression of first pain and central summation of second pain evoked by noxious heat pulses. Pain 3:57-68.

Price DD, Milling LS, Kirsch I, Duff A, Montgomery GH, Nicholls SS (1999) An analysis of factors that contribute to the magnitude of placebo analgesia in an experimental paradigm. Pain 83:147-156.

Sawamoto N, Honda M, Okada T, Hanakawa T, Kanda M, Fukuyama H, Konishi J, Shibasaki H (2000) Expectation of pain enhances responses to nonpainful somatosensory stimulation in the anterior cingulate cortex and parietal operculum/posterior insula: an event-related functional magnetic resonance imaging study. J Neurosci 20:7438-7445.

Stewart-Williams S, Podd J (2004) The placebo effect: dissolving the expectancy versus conditioning debate. Psychol Bull 130:324-340.

Talairach J, Tournoux P (1988) A coplanar stereotactic atlas of the human brain. Stuttgart: Thieme.

Tracey I, Ploghaus A, Gati JS, Clare S, Smith S, Menon RS, Matthews PM (2002) Imaging attentional modulation of pain in the periaqueductal gray in humans. J Neurosci 22:2748-2752.

Valet M, Sprenger T, Boecker H, Willoch F, Rummeny E, Conrad B, Erhard P, Tolle TR (2004) Distraction modulates connectivity of the cingulofrontal cortex and the midbrain during pain-an fMRI analysis. Pain 109:399-408.

Villemure C, Bushnell MC (2002) Cognitive modulation of pain: how do attention and emotion influence pain processing? Pain 95:195-199.

Voudouris NJ, Peck CL, Coleman G (1990) The role of conditioning and verbal expectancy in the placebo response. Pain 43:121-128.

Wager TD, Rilling JK, Smith EE, Sokolik A, Casey KL, Davidson RJ, Kosslyn SM, Rose RM, Cohen JD (2004) Placebo-induced changes in fMRI in the anticipation and experience of pain. Science 303:1162-1167.

Wiberg M, Westman J, Blomqvist A (1987) Somatosensory projection to the mesencephalon: an anatomical study in the monkey. J Comp Neurol 264:92-117.

Willis WD, Westlund KN (1997) Neuroanatomy of the pain system and of the pathways that modulate pain. J Clin Neurophysiol 14:2-31.

Yezierski RP (1988) Spinomesencephalic tract: projections from the lumbosacral spinal cord of the rat, cat, and monkey. J Comp Neurol 267:131-146.

Zambreanu L, Wise RG, Brooks JC, Iannetti GD, Tracey I (2005) A role for the brainstem in central sensitisation in humans. Evidence from functional magnetic resonance imaging. Pain 114:397-407. 